\title{
THE SURGICAL IMPORTANCE OF A PERSISTENT LEFT SUPERIOR VENA CAVA*
}

\author{
BY \\ ELLIOTT S. HURWITT $\dagger$ \\ From the Surgical Division, the Montefiore Hospital, New York City
}

\begin{abstract}
A left superior vena cava may be present simultaneously with a normal right superior vena cava, or, even more infrequently, as the only superior vena cava. The surgical importance of a persistent left superior vena cava varies depending on its termination into the right or left atrium, as well as the nature and severity of any associated cardiovascular anomalies.
\end{abstract}

\section{Left Superior Vena Cava Entering Right Atrium.} This is not a particularly uncommon anomaly, the vein terminating either directly into the right atrium, or indirectly by way of the coronary sinus. The embryology and frequency of this configuration have been reviewed in a previous communication (Hurwitt, Escher and Citrin, 1955). Since anomalies of the heart and great vessels tend to occur in association, one may encounter a left superior vena cava draining into the right atrium in the process of other operations, such as in the correction of a patent ductus arteriosus. Under such circumstances the anatomical course of the left superior vena cava is such that it does not interfere with the stated operation, and the vein usually has no surgical importance.

With the advent of open heart surgery, however, employing either cardiopulmonary by-pass or hypothermia, a left superior vena cava assumes real surgical significance, regardless of whether it drains into the right or left atrium. Failure to identify and mobilize this vessel, with temporary occlusion for hypothermia and cannulation or occlusion during cardiopulmonary by-pass, may seriously compromise the procedure. The necessity for this manoeuvre has been described in a number of articles, including one from this department (Robinson, Glotzer, Gilbert, Escher and Hurwitt, in press).

\footnotetext{
* Presented at the Fifth Annual Meeting, the British Association of Paediatric Surgeons, London, July 23, 1958.

$\dagger$ Aided by a grant from the New York Heart Association.
}

Left Superior Vena Cava Entering Left Atrium. As a contributing cause of cyanosis, the importance of drainage of any systemic vein into the left auricle has usually been overshadowed by the severity of associated cardiac defects. Subsequent to the summary of the reported cases (Hurwitt et al., 1955), two additional such patients have come to surgery at this hospital.

\section{Case Histories}

\section{Uncorrected Lesions}

Case 1. A $4 \mathrm{lb}$. premature infant, one of male twins, was brought to the hospital on January 21, 1957, with complete ectopia cordis (Fig. 1) and severe cyanosis and dyspnoea; the twin was normal. A covering of skin was fashioned over the heart, and the baby died on the evening of the operation. A left superior vena cava entering the left atrium was only one of the eight major anomalies of the heart and great vessels found at autopsy. A normal right superior vena cava was also present. A motion picture and complete description of this case have been prepared (Hurwitt and Lebendiger, in press).

Case 2. Severe cyanosis and nearly fatal episodes of syncope were the presenting problems in this 18-monthold girl. Investigation in another hospital had established the presence of both a right and left superior vena cava and a levocardia in the presence of a situs inversus. abdominalis. On June 17, 1958, an attempt was made to construct a Blalock shunt between the left subclavian artery and the left pulmonary artery, but irreversible cardiac arrest developed during mobilization of the pulmonary artery. Post-mortem examination disclosed an essentially bilocular heart, with the left superior vena cava draining into the left side of a chamber that was practically a common auricle. Splenic agenesis, described in several such cases, was not present.

\section{Corrected Lesions}

Even when severe associated anomalies of the heart are present, however, interruption of the flow of systemic venous blood into the left auricle may be beneficial, with or without attempted correction of the other lesions. Diaz and Anido (1949) reported significant improvement 


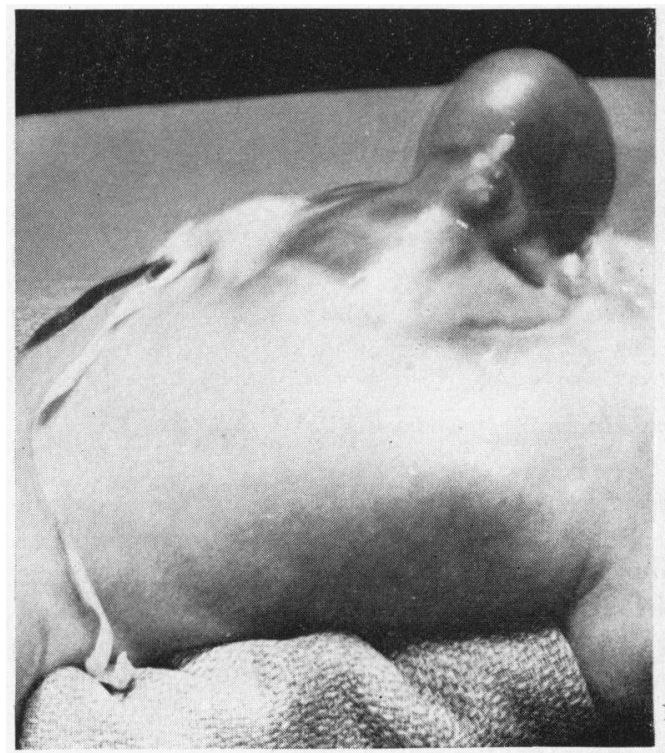

FIG. 1. Case 1. Photograph and diagram of ectopia cordis, with bilateral superior venae cavae.

in a 7-year-old tetralogy case following ligation of a left superior vena cava entering the left auricle, without any other procedure. Lessening of cyanosis was similarly described in an 8-year-old with Eisenmenger's complex, by Feindt and Hauch (1953). Ekstrom (personal communication) supplemented construction of a Blalock shunt in a 12-year-old with tetralogy of Fallot, situs inversus totalis and double superior venae cavae, by ligating the anomalous vena cava as it entered the arterial auricle.

Obviously the outlook is much more favourable when the left superior vena cava entering the left auricle constitutes the major cause of cyanosis, or when any concomitant intracardiac defects may also be amenable to correction. Examples of each of these situations have now come to operation at this hospital.

Case 3. This has previously been reported in detail (Hurwitt et al., 1955). Surgical relief of cyanosis in an 8-year-old girl, due to entrance of a left superior vena cava into the left auricle, was accomplished by intrapericardial ligation of the left superior vena cava on December 29, 1954 (Fig. 2). The venous return to the heart after the procedure is depicted in Fig. 3. Post-operatively there was marked improvement in clubbing of the fingers, cyanosis. dyspnoea, appetite, and exercise performance. Slight peripheral arterial oxygen unsaturation persisted, presumably related to an atypical moderate hypoplasia of the posteriorly placed main pulmonary artery.

Case 4. A 10-year-old negro girl with acyanotic congenital heart disease was found on cardiac catheteriza-

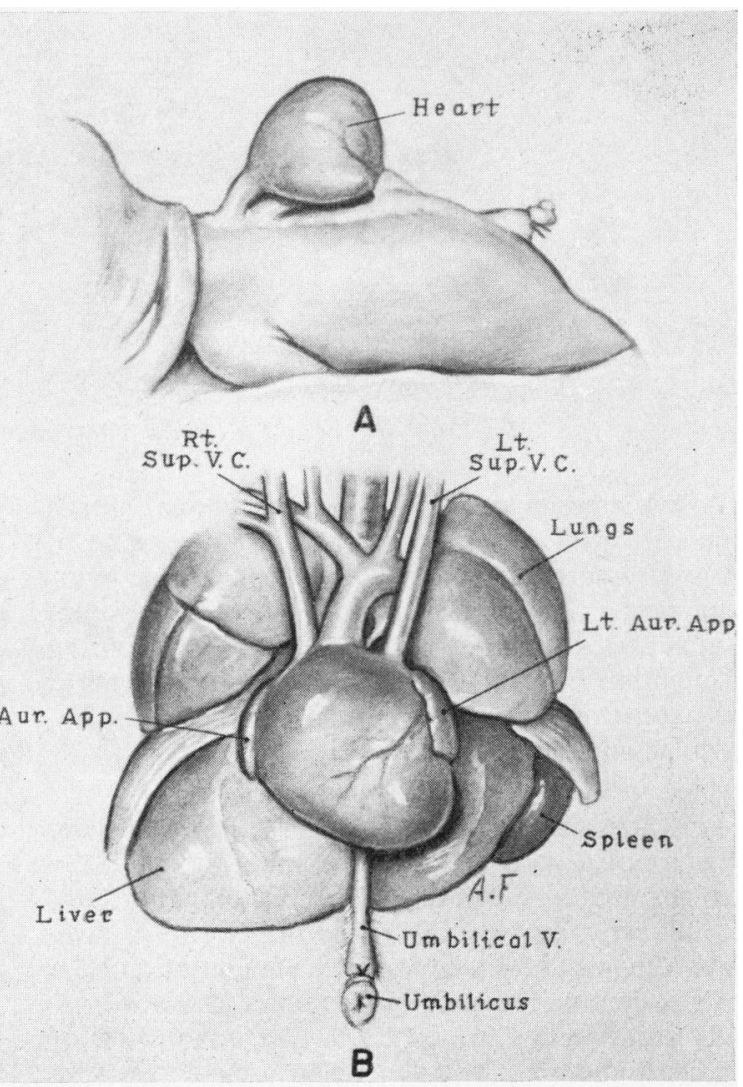

tion to have an atrial septal defect with a significant flow from left to right and a left superior vena cava. Operation, under hypothermia, was performed on July 1 , 1957, through an anterior thoracic incision, entering the right pleural cavity in the fourth interspace and the left pleural cavity in the third interspace, with transverse division of the sternum. On the right there was a small superior vena cava with a normal azygos tributary. The left superior vena cava was considerably larger, was joined by a large hemiazygos vein, and entered the left atrium anteriorly to the entrance of the left pulmonary veins. Digital exploration of the right auricle confirmed the presence of a large atrial septal defect, and oxygen determinations from both auricles showed complete admixture of the blood at this level under the operative conditions (Fig. 4a).

At a body temperature of $88^{\circ} \mathrm{F}$. the inferior and the two superior venae cavae were occluded for six and a half minutes while the right auricle was opened and an atrial septal defect measuring $4 \mathrm{~cm}$. in length and $2 \frac{1}{2} \mathrm{~cm}$. in width was repaired by a continuous silk suture. While the right auricle now contained blood with a venous oxygen saturation, blood from the left auricle at the site of insertion of the left superior vena cava was also highly 

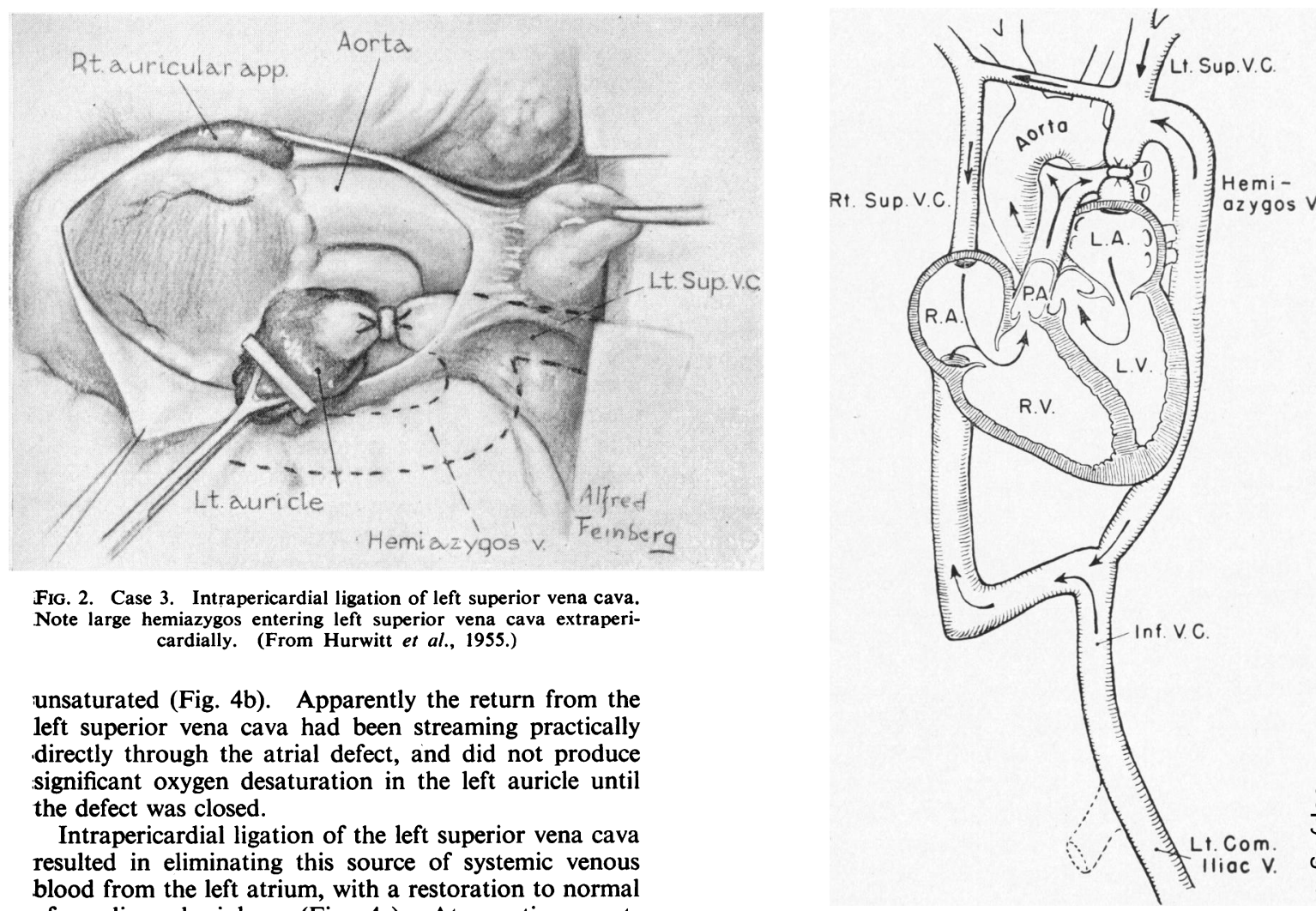

FIG. 2. Case 3. Intrapericardial ligation of left superior vena cava. Note large hemiazygos entering left superior vena cava extrapericardially. (From Hurwitt et al., 1955.)

unsaturated (Fig. 4b). Apparently the return from the left superior vena cava had been streaming practically directly through the atrial defect, and did not produce significant oxygen desaturation in the left auricle until the defect was closed.

Intrapericardial ligation of the left superior vena cava resulted in eliminating this source of systemic venous blood from the left atrium, with a restoration to normal of cardiac physiology (Fig. 4c). At no time postoperatively was there any evidence of oedema, cyanosis, or increased venous pressure, confirming the presence of adequate communicating channels between the two superior vena caval systems. The child was re-examined

Fig. 3. Case 3. Diagrammatic reconstruction of vascular anomalies and ligation of left superior vena cava. (From Hurwitt $e t$ al., 1955.) Both figures are reproduced by courtesy of C. V. Mosby Co., St. Louis, Mo.

OXYGEN SATURATION

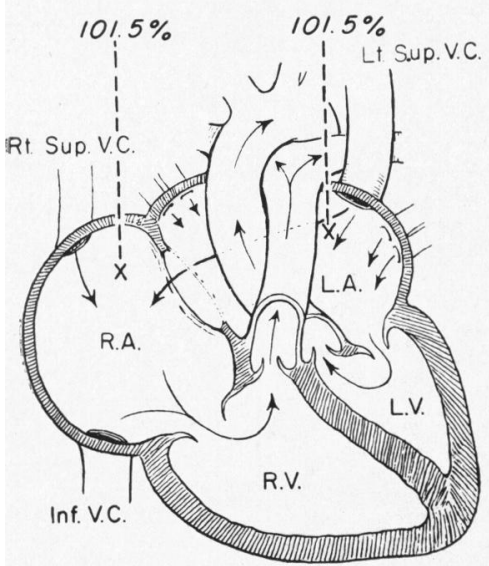

OXYGEN SATURATION

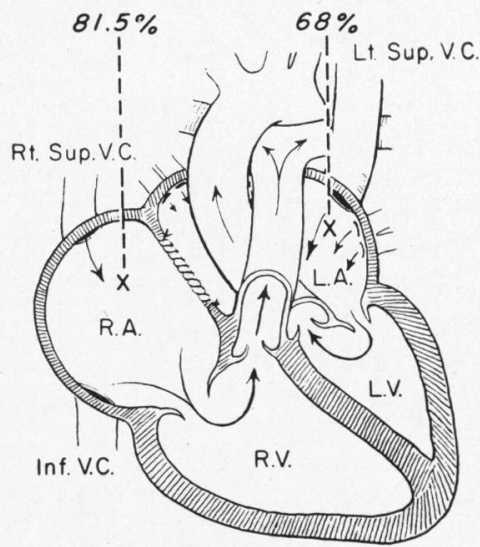

OXYGEN SATURATION

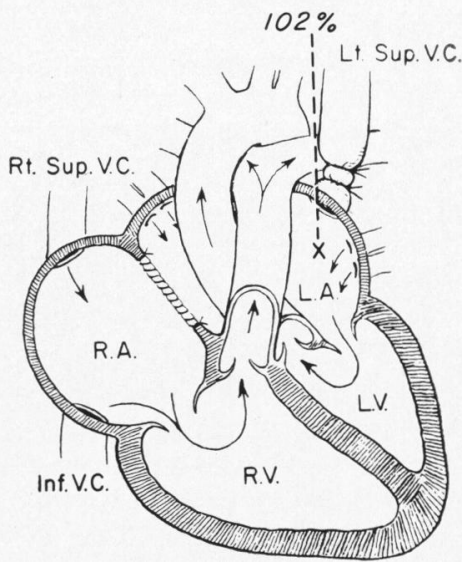

FIG. 4a, b, c.-Case 4. Status at operation: $(a)$, initially; $(b)$, after closure of the atrial septal defect; and (c), after ligation of the left superior vena cava. 
one year after operation and found to be in excellent health.

\section{Comment}

Drainage of any systemic vein into the left atrium contributes to the cyanotic state, and as such merits consideration for surgical correction; the only categorical contra-indication would be atresia of the tricuspid valve. The anomalous vein will usually be a superior vena cava, although the inferior vena cava (Gardner and Cole, 1955), coronary sinus (Mankin and Burchell, 1953), and levo-atriocardinal veins (Gould, 1953) have also been reported terminating in the left auricle.

The degree of improvement following ligation of a left superior vena cava will be determined by the severity of any uncorrected associated lesions. It may be substantial, however, even in the presence of complex defects of the tetralogy type (Gould, 1953; Gardner and Cole, 1955; Hurwitt et al., 1955). When the associated anomalies are also corrected, as in Case 4, or when no major concomitant abnormalities are found, the result may be excellent. Cases have been described with no associated septal defects (Potter, 1948; Gardner and Cole, 1955; Peel, Semple, Kelly and Blum, 1956; Tuchman, Brown, Huston, Weinstein, Rowe and Crumpton, 1956), and none was found in our Case 3. When the flow from the left superior vena cava is streaming almost directly through the atrial septal defect, significant arterial oxygen unsaturation may not be detected until the defect is closed, as in Case 4. Occlusion of the left superior vena cava then restores the situation to normal.

A disadvantage of approaching repair of an atrial septal defect through an incision limited to opening the right pleural cavity is the possibility of overlooking the presence of a left superior vena cava. Even in the hands of those who employ 'closed' techniques for these operations, the opportunity for correcting a left superior vena cava entering the left auricle would be missed. Most surgeons currently close atrial septal defects under direct vision with 'open' techniques. If venous inflow occlusion with hypothermia is practised, failure to take a left superior vena cava into account may result both in excessive blood loss and poor visualization of the defect, regardless of which atrium receives the anomalous vein; the same considerations apply equally to cardiopulmonary by-pass. For these reasons either a vertical sternum-splitting incision or a bilateral anterior thoracotomy with division of the sternum are preferable to right thoracotomy alone.

Interruption of a left superior vena cava entering the left auricle must be performed intrapericardially in order also to divert the flow from the hemiazygos vein to the right side (Figs. 2, 3). The interruption may be accomplished simply by ligation, as in the reported cases, or by division. If a solitary left superior or inferior vena cava were found entering the left atrium, transplantation to the right side would be necessary, either by direct suture or by a graft. One unsuccessful attempt to re-route blood in a solitary left superior vena cava from the left to the right auricle has been reported by Tuchman $e t$ al. (1956). When bilateral superior venae cavae are present, apparently the communicating veins across the mediastinum or through the azygos system are usually adequate, so that closure of the left superior vena cava results in shunting the blood to the right side, rather than the syndrome of superior vena caval compression. When such communications are absent, however, as suggested in the report by Peel et al. (1956), interruption of the left superior vena cava should be supplemented by an anastomosis to the right superior caval system.

\section{Summary}

The surgical importance of a left superior vena cava has been analysed.

Regardless of whether the left superior vena cava drains into the right or left atrium, it must be either occluded or cannulated during open heart surgery with either hypothermia or cardiopulmonary by-pass.

Any systemic vein entering the left auricle may be a major or contributing cause of cyanosis, and should be corrected, except in the presence of tricuspid atresia.

Data are presented on four cases of left superior vena cava entering the left atrium, with surgical correction in two instances, one of which has been reported previously.

Ligation of a left superior vena cava entering the left auricle and closure of a large atrial septal defect were accomplished in a 10-year-old girl.

This represents the fifth reported case of relief of cyanosis following interruption of a left superior vena cava terminating in the left auricle.

REFERENCES

Ekstrom, G. (1954). Personal communication.

Feindt, H. R. and Hauch, H. J. (1953). Z. Kreisl.-Forsch., 42, 53. Gardner, D. L. and Cole, L. (1955). Brit. Heart J., 17, 93.

Gould, S. E. (1953). Pathology of the Heart, pp. 49i, 496. Charles C. Thomas, Springfield, Illinois.

Hurwitt, E. S., Escher, D. J. W. and Citrin, L. I. (1955). Surgery, 38, 903 .

and Lebendiger, A. A.M.A. Arch. Surg. In the press. Presented at the Sixth Scientific Meeting, the North American Chapter of the International Cardiovascular Society, San Francisco, June 21, 1958.

Mankin, H. T. and Burchell, H. B. (1953). Proc. Mayo Clin., 28, 463. Peel, A. A. F., Semple, T., Kelly, J. C. and Blum, K. (1956). Scot. med. J., 1, 83 .

Potter, E. L. (1948). Arch. Path. (Chicago), 46, 87.

Robinson, G., Glotzer, P., Gilbert, M., Escher, D. J. W. and Hurwitt E. S. Amer. J. Cardiol. In the press. Presented in the New
. Ament, York Society for Thoracic Surgery, May 9, 1958.

Rodiguez Diaz, A. and Anido, H. (1949). Dis. Chest, 15, 684.

Tuchman, H., Brown, J. F., Huston, J. H., Weinstein, A. B., Rowe, G. G. and Crumpton, C. W. (1956). Amer. J. Med., 21, 481' 